\title{
Spatial variation in prostate cancer survival in the Northern and Yorkshire region of England using Bayesian relative survival smoothing
}

\author{
L Fairley*, ${ }^{*}$ D Forman ${ }^{1,2}$, R West ${ }^{3}$ and S Manda ${ }^{4}$ \\ 'Northern and Yorkshire Cancer Registry and Information Service, St James's Institute of Oncology, St James's University Hospital, Level 6, Bexley Wing, \\ Beckett Street, Leeds LS9 7TF, UK; ${ }^{2}$ Cancer Epidemiology Group, Division of Epidemiology and Biostatistics, University of Leeds, St James's Institute of \\ Oncology, St James's University Hospital, Bexley Wing, Beckett Street, Leeds LS9 7TF, UK; ${ }^{3}$ Biostatistics Unit, Division of Epidemiology and Biostatistics, \\ University of Leeds, Worsley Building, Leeds LS2 9JT, UK; ${ }^{4}$ Biostatistics Unit, South African Medical Research Council, Private Bag X385, Pretoria 000 I, \\ South Africa
}

Primary Care Trust (PCT) estimates of survival lack robustness as there are small numbers of deaths per year in each area, even when incidence is high. We assess PCT-level spatial variation in prostate cancer survival using Bayesian spatial models of excess mortality. We extracted data on men diagnosed with prostate cancer between 1990 and 1999 from the Northern and Yorkshire Cancer Registry and Information Service database. Models were adjusted for age at diagnosis, period of diagnosis and deprivation. All covariates had a significant association with excess mortality; men from more deprived areas, older age at diagnosis and diagnosed in 1990-1994 had higher excess mortality. The unadjusted relative excess risks (RER) of death by PCT ranged from 0.75 to I.66. After adjustment, areas of high and low excess mortality were smoothed towards the mean, and the RERs ranged from 0.74 to I.49. Using Bayesian smoothing techniques to model cancer survival by geographic area offers many advantages over traditional methods; estimates in areas with small populations or low incidence rates are stabilised and shrunk towards local and global risk estimates improving reliability and precision, complex models are easily handled and adjustment for covariates can be made. British Journal of Cancer (2008) 99, I786- |793. doi:I0.1038/sj.bjc.6604757 www.bjcancer.com

Published online 4 November 2008

(C) 2008 Cancer Research UK

Keywords: Bayesian analysis; spatial models; relative survival; prostate cancer

Five-year relative survival rates from prostate cancer in England and Wales have increased from $43 \%$ for men diagnosed during $1986-1990$ to $68 \%$ for men diagnosed in 1996-1999. The deprivation gap in survival also increased over this time period to a difference of $7 \%$ between men from the most affluent areas compared with men from the most deprived areas (Coleman et al, 2004). This increase in survival reflects the increased use of prostate-specific antigen (PSA) testing, which has led to the diagnosis of many asymptomatic prostatic cancers that would never have been diagnosed in life (Brewster et al, 2000; Evans and Moller, 2003).

Survival from prostate cancer is known to vary among different geographic regions. The EUROCARE study found that there was considerable variation in survival between countries, with England having one of the lowest survival rates (Post et al, 1998; EUROCARE, 2003; Berrino et al, 2007). Significant geographical variation in prostate cancer survival has also been observed in Australia (Yu et al, 2004), the Nordic Countries (Dickman et al, 1997) and the United States (Farrow et al, 1996). Within the Northern and Yorkshire regions the 5-year relative survival from

*Correspondence: L Fairley; E-mail: lesley.fairley@nycris.leedsth.nhs.uk Received 9 June 2008; revised 25 September 2008; accepted I October 2008; published online 4 November 2008 prostate cancer has been shown to vary by cancer network from 62 to $72 \%$ (NYCRIS, 2004).

Most regional analyses of survival estimate rates for each region separately and compare them to assess the regions that are statistically different from each other. This leads to problems with multiple testing; if there are many areas some will be found to be statistically significantly different through chance alone. In addition, areas with sparse data will have large confidence intervals and will produce unstable estimates of survival rates. Furthermore, independently estimating the survival rates for each area makes no use of data in the surrounding areas. The result is that the areaspecific estimates of survival rate have low power and validity, in addition to poor precision.

Most of these problems can be overcome by statistical smoothing techniques, which by borrowing and sharing data across areas, produce more reliable estimates of risk. In particular, estimates are stabilised and shrunk towards average values; thus overcoming the problems of areas with small populations or low incidence rates. Of the many methods available, Bayesian methods are commonly used to generate smoothed estimates of risk in cancer relative survival ( $\mathrm{Yu}$ et al, 2004). Bayesian methods are proving useful and applicable because of the ease with which prior information is included in the analyses; moreover, complex and realistic models are easily handled as opposed to traditional methods. In using a Bayesian approach, we obtain posterior 
distributions of all the model parameters and their functions such as relative contributions of the unstructured and structured spatial effect variations.

There has been a trend in the last few years within epidemiology to perform spatial variation of diseases risk at the smallest geographical area such as electoral wards. However, in the case of cancer survival where there is a need to calculate expected deaths in follow-up intervals (usually yearly intervals), the observed deaths are very sparse, which makes the assumptions on which estimating methods are based invalid. (Rachet and Coleman, 2004) Cancer survival estimates are not routinely produced at Primary Care Trust (PCT) levels, as PCT level estimates of survival are insufficiently robust. Rachet and Coleman (2004) found that annual estimates of relative survival might not be reliable for PCTs because of small numbers of deaths, even in cancers where the incidence is high. That report was based on the PCTs prior to the most recent reorganisation in October 2006; however, even the newly configured PCTs, although much larger than the previous ones, are still too small to be used as geographic units for monitoring cancer survival. (Ellis et al, 2007) Using Bayesian smoothing methods to estimate survival for each PCT will overcome many of these problems by borrowing and sharing data across areas overcoming the problems with areas with low incidence or few deaths. We have used PCT (using pre October 2006 boundaries) as our level of analysis. Moreover PCTs are a logical unit to use as they are the focus of most of the NHS cancer policy and responsible for delivering care to patients and commissioning services within that area. It is also important not only to provide an estimate for each PCT, but also to see how these estimates relate to other PCTs.

We set out to apply full Bayesian smoothing techniques using hierarchical models to assess PCT-level spatial variation in prostate cancer relative survival. By, taking into account both local and global risk smoothing, these methods produce more reliable, precise and robust estimators for PCT-specific variation in prostate cancer relative survival. We also investigated if the spatial variation in survival can be explained by socio-demographic risk factors, age, deprivation and period of diagnosis.

\section{MATERIALS AND METHODS}

\section{Data}

The Northern and Yorkshire Cancer Registry and Information Service (NYCRIS) is a population-based cancer registry covering a total population of 6.7 million (at the 2001 Census). The analysis in this paper is based on the Primary Care Trust boundaries that were effective until October 2006. Within the NYCRIS region there were 44 PCTs during this time period. The average size of the PCT populations in 2001 was just under 150000 , this ranged from just under 70000 in Eden Valley to about 307000 in Northumberland.

All prostate cancer (ICD10 C61) cases diagnosed in the NYCRIS region between 1990 and 1999 were extracted from the registry database $(n=22042)$. These cases were then followed up for death until 31 December 2004. There were 492 cases that were Death Certificate Only and no date of diagnosis was available for these cases so they were excluded from the analysis. Cases aged over 100 at diagnosis and cases that were multiple primaries were also excluded from the analysis (2097 cases). This left 19453 cases to be included in the survival analysis but a further 45 cases had the date of diagnosis the same as the date of death and therefore had zero survival time and were also excluded from the analysis. The resulting study population consisted of 19408 men diagnosed with prostate cancer between 1990 and 1999 and followed up till the end of 2004.

For each patient we also obtained their age at diagnosis, ward and PCT of residence at diagnosis and year of diagnosis. Age was grouped into four different categories - 15-59 years, 60-69 years, 70-79 years and $80+$ years. Deprivation was measured at the ward level and derived from the Index of Multiple Deprivation 2004 Income domain scores (Nobel et al, 2004); each patient was assigned a deprivation quintile based on their ward of residence. Year of diagnosis was split into two time periods 1990-1994 and $1995-1999$.

\section{Statistical methods}

Relative survival Relative survival is the preferred method of survival analysis for population-based cancer survival. The cause of death is not reliably reported for all cancer patients and even with access to medical records it is difficult to classify each patient's death into one of the two categories 'entirely due to cancer' or 'entirely unrelated to cancer'. Therefore, rather than using cause-specific survival, relative survival is used when calculating survival from cancer. Relative survival is the ratio of the observed proportion surviving in a group of patients to the expected proportion that would have survived in a comparable group of people (with for example, the same distribution by age, sex and the geographical area) from the general population. The major advantage of this measure is that information on cause of death is not required and it provides a measure of the excess death rate experienced by patients diagnosed with cancer, irrespective of whether the excess is directly or indirectly attributable to the cancer (Ederer et al, 1961; Hakulinen and Tenkanen, 1987).

Modelling relative survival To model the relative survival so we could include covariates in the analysis we fitted a Poisson model for relative survival as described in Dickman et al (2004) and Pohar and Stare, (2006) assuming an additive hazard model. The hazards are assumed to be constant within pre-specified sub intervals of follow-up time (that is, piecewise constant hazards). In this study, we have used follow-up intervals of 1 year. A set of indicator variables was constructed, one indicator variable for each interval, and incorporated into the covariate vector. Our primary interest is in the excess hazard component, which is assumed to be an exponential function of the covariates, written as $\exp (z \beta)$ where $\mathrm{z}$ is the vector of predictor variables with the associated vector of coefficients in $\beta$, that is, the Cox model. Parameters representing the effect in each follow-up time interval are estimated in the same way as parameters representing the effect of, for example, age or sex. In our models we have age at diagnosis (four categories) deprivation quintile (five categories) and period of diagnosis (two categories) as covariates.

We assume that patients are grouped into $k$ strata based on a combination of the relevant predictors, that is, one stratum for each combination. Furthermore, assume that there are $i$ intervals of follow up. Following Dickman et al (2004) let the number of deaths, $\mathrm{d}_{k i}$, be distributed as a Poisson distribution, $\mathrm{d}_{k i} \sim$ Pois$\operatorname{son}\left(\mu_{k i}\right)$ where $\mu_{k i=} \lambda_{k i} y_{k i}$ and $y_{k i}$ is person time at risk for the observations in stratum $k$ in the interval $i$. If we denote $\mathrm{d}_{k i}{ }^{*}$ as the expected number of deaths in stratum $k$ and interval $i$, then

$$
\ln \left(\mu_{k i}-d_{k i}^{*}\right)=\ln \left(y_{k i}\right)+z \beta
$$

Note that $d_{k i}{ }^{*}$ is the expected number of deaths (due to causes other than the cancer of interest and estimated from general population mortality rates). This is a generalised linear model with outcome $\mathrm{d}_{k i}$ with a Poisson error structure and a link function $\ln \left(\mu_{k i}-\mathrm{d}_{k i}{ }^{*}\right)$ with offset $\ln \left(y_{k i}\right)$. This is not a standard link function so fitting the model requires software which allows user-defined link functions, in our analysis we fitted it in WinBUGS (Spiegelhalter et al, 2004). The exponentiated parameter estimates have an interpretation as excess hazard ratios also known as relative excess risk (RER). 
We used the Stata macro strs, written by Paul Dickman (available from http://www.pauldickman.com) to calculate expected survival using the Ederer II method (Dickman et al, 2008), using lifetables derived specifically for the Northern and Yorkshire region. Each individual's follow up time was censored at 5 years. For each individual we estimated the expected number of deaths in each follow up interval and also the person time at risk as well as an indicator of whether or not they died in that interval. We then aggregated these values for each PCT for each combination of covariates in each follow up period (4 age groups $\times 5$ deprivation groups $\times 2$ periods of diagnosis $=40$ combinations of covariate groups (5 follow up periods and 44 PCTs)).

Bayesian spatial models We used Bayesian spatial models to fit both local and global smoothed estimates of relative excess risk across the 44 PCTs (Besag et al, 1991). This was done by modifying (1) to include PCT random effects

$$
\ln \left(\mu_{k i}-d_{k i}^{*}\right)=\ln \left(y_{k i}\right)+z \beta+u_{l}+v_{l}, \quad l=1, \mathrm{~K} \ldots, 44
$$

where $u_{1}$ and $v_{1}$ are the unstructured and structured random effects, which are assigned a normal and a conditional autoregressive prior distribution. The former smoothes the relative risks towards a global value, whereas the latter towards the mean risk of the neighbouring areas, with variance inversely proportional to number of neighbours (Besag et al, 1991). Unstructured heterogeneity variance $\left(\sigma^{2}\right)$ and conditional spatial variance $\left(\theta^{2}\right)$ are each given $\gamma$ prior distributions with scale parameter 0.5 and shape parameter 0.001 .

\section{Relative contributions of spatial and unstructured heterogeneity in the convolution model}

Unstructured heterogeneity variance $\left(\sigma^{2}\right)$ and spatial variance $\left(\theta^{2}\right)$ are not directly comparable, $\sigma^{2}$ reflects marginal variability of the unstructured random effects between areas whereas, $\theta^{2} / n_{1}$ reflects conditional variance of the spatial effect of area $l$ conditional on the values of the neighbouring spatial effects, where $n_{1}$ is the number of neighbours of area $l$. Therefore, we have to estimate the marginal spatial variance empirically where,

$$
\theta_{\text {marginal }}^{2}=\sum_{l}\left(v_{l}-\bar{v}\right)^{2} /(I-1)
$$

From this we can then estimate the relative contributions of spatial and unstructured heterogeneity. We define the spatial fraction
(Frac spatial) as

$$
\operatorname{Frac}_{\text {spatial }}=\theta_{\text {marginal }}^{2} /\left(\theta_{\text {marginal }}^{2}+\sigma^{2}\right)
$$

If the spatial fraction is close to 1 then the spatial heterogeneity dominates and if the spatial fraction is close to 0 then the unstructured heterogeneity dominates. Spatially structured variance measures the amount of local smoothing whereas the unstructured variance measures global smoothing in the PCT-relative risks. In the model where possible risk factors are not included, these show the overall variation, otherwise they show excess variation above that captured by the included risk factors. We compared this spatial fraction after including different variables in the model to see how much of the geographical variation between PCTs can be explained by the risk factors.

We fitted four different models, one unadjusted (only including the follow up interval) one adjusted for each covariate in turn and one fully adjusted model, which included all covariates. We used the Deviance Information Criterion (DIC) to asses the model fit (Spiegelhalter et al, 2002) where the model with the smaller DIC is better supported by the data. We used a 'burn-in' period of 40000 iterations and then ran a further 40000 iterations to obtain the posterior estimates. We report the median and 95\% credible intervals for the posterior summaries. For comparison we also fitted the models in Stata to obtain RERs for each PCT using a classical Poisson model.

\section{RESULTS}

Table 1 shows the distribution of the demographic variables in the study population, with the corresponding 5-year survival and Figure 1 shows the Kaplan-Meier survival curves up to 5 years of follow up by each of the demographic variables. Over $90 \%$ of the men were aged 60 and over at diagnosis with $43 \%$ of them aged $70-79$ years. There were more men from the more deprived areas than the most affluent areas (15\% of men were in the most affluent deprivation quintile compared with $24 \%$ of men in the most deprived quintile). Slightly more men were diagnosed in the later time period $(56 \%)$ than in the earlier time period $(44 \%)$. The overall Kaplan-Meier 5-year survival rate for men diagnosed with prostate cancer in the NYCRIS region was $41 \%$ (95\% CI $41-42$ ). Survival decreased as age at diagnosis increased; men aged 15-59 years at diagnosis had a 5 -year survival rate of $62 \%$ whereas it was only $19 \%$ for men aged 80 and over at diagnosis. There was a

\begin{tabular}{|c|c|c|c|c|}
\hline Variable & $\mathbf{N}$ & $\%$ & 5-year Kaplan-Meier survival (\%) & $95 \% \mathrm{Cl}$ \\
\hline $\begin{array}{l}\text { Period of diagnosis } \\
1990-1995 \\
1996-2000\end{array}$ & $\begin{array}{r}8556 \\
10852\end{array}$ & $\begin{array}{l}44.08 \\
55.92\end{array}$ & $\begin{array}{l}35.0 \\
46.1\end{array}$ & $\begin{array}{l}(34.0,36.0) \\
(45.2,47.1)\end{array}$ \\
\hline
\end{tabular}
difference of about 8 percentage points in the 5-year survival of

Table I Demographic characteristics of the study population and Kaplan-Meier 5-year survival rates 

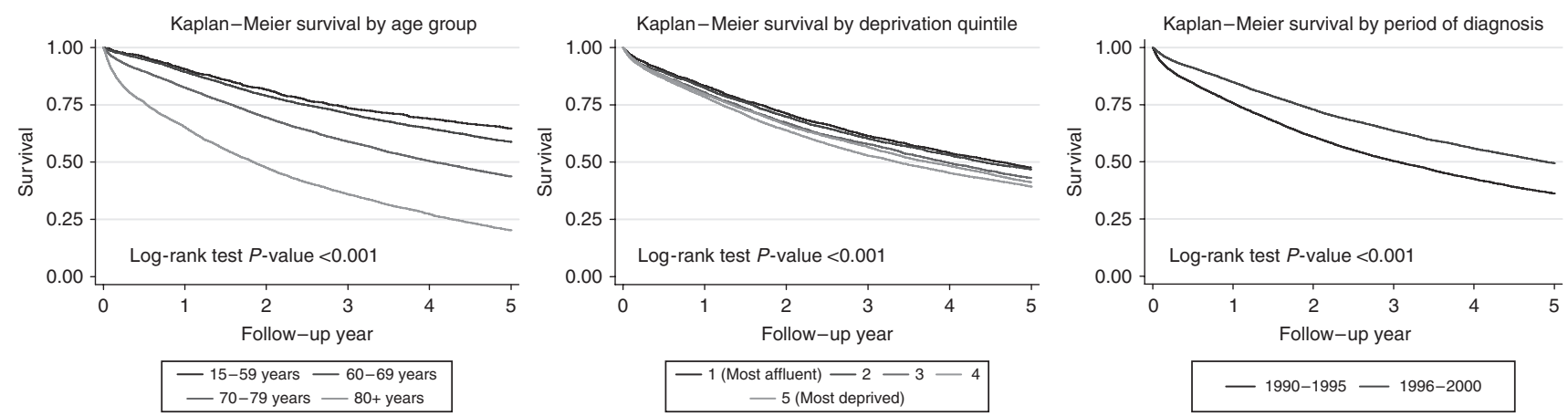

Figure I Kaplan-Meier Survival curves by each covariate.

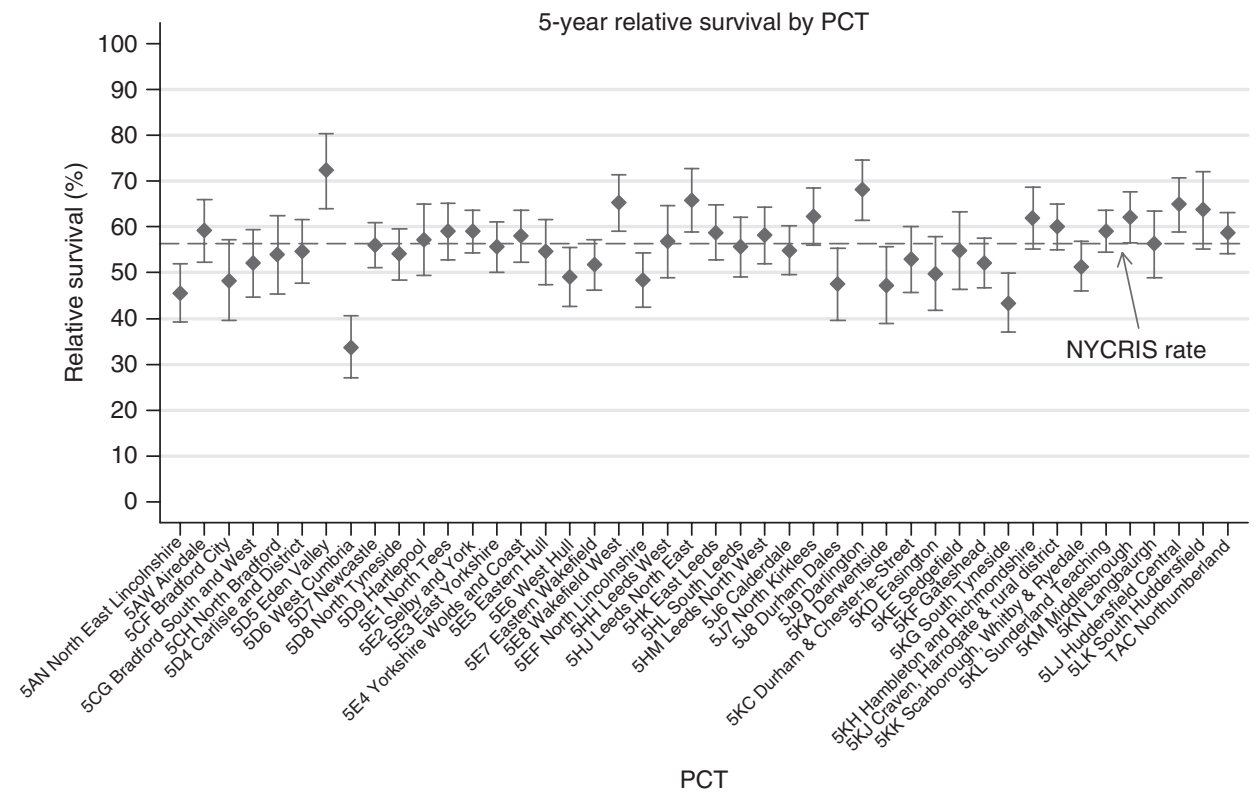

Figure 2 Relative survival by PCT.

men from the most affluent areas compared with those from the most deprived (46 and 38\% respectively). The 5-year survival rate of men diagnosed between 1990 and 1994 was significantly lower than for men diagnosed between 1995 and 1999 (35 and 46\% respectively).

The graph in Figure 2 shows the 5-year relative survival rates by PCT. The overall survival rate in the NYCRIS region was 56\% (95\% CI 55.4-57.3), this ranged from $34 \%$ in West Cumbria PCT to $72 \%$ in Eden Valley PCT. There were five PCTs that had a significantly higher relative survival rate than the NYCRIS rate and five PCTs that had a significantly lower relative survival rate.

Table 2 shows the results from the Bayesian Poisson models of excess mortality for the unadjusted, univariate and fully adjusted models. The last column also shows the results from the classical Poisson regression and the estimated relative excess risks are very similar to the Bayesian estimates. Comparing the Bayesian models the DIC for the fully adjusted model is about 730 points lower than the unadjusted model and substantially lower than each of the univariate models suggesting that the fully adjusted spatial model is better supported for the data (Table 3). The fixed effects estimates in the univariate and fully adjusted models are similar. From the fully adjusted model the excess mortality associated with a diagnosis of cancer is 2.27 times higher for men aged 80 and over at diagnosis compared with men aged 15-59 years at diagnosis
(95\% CI 2.02-2.55). There was also significant deprivation effect, with an RER of 1.43 in the most deprived areas compared with the most affluent areas (95\% CI 1.28-1.58). Men diagnosed later had a significantly lower excess mortality rate than men diagnosed in the earlier period $(\mathrm{RER}=0.67,95 \%$ CI $0.64-0.71$ ).

Figure 3 shows the unadjusted and fully adjusted RER for each PCT. As expected many of the PCTs with relative survival rates less than the NYCRIS rate had RERs higher than 1 and PCTs with relative survival rates higher than the NYCRIS rates had RERs less than 1. The unadjusted RER range from 0.76 to 1.66; seven PCTs have significantly higher RERs compared with the overall NYCRIS region and seven PCTs have significantly lower RERs. The fully adjusted RERs range from 0.74 to 1.49 , and from the graph we can see that the fully adjusted model shrinks the RERs towards the baseline value of 1 . The number of PCTs significantly different from the baseline is reduced to four PCTs with significantly higher RERs and four PCTs with significantly lower RERs.

Table 4 shows descriptive statistics comparing the adjusted RERs for the PCTs using a Bayesian and classical approach. As expected under Bayesian hierarchical spatial analysis, the PCT-relative excess risk estimates are less dispersed around the median of 1 than the classical estimates.

In the unadjusted model $70 \%$ of the variance between PCTs was due to spatial effects although the $95 \%$ credible interval for this 
Table 2 Covariate fixed effects of estimates of the relative excess risk of death

\begin{tabular}{|c|c|c|c|c|c|c|}
\hline \multirow[b]{2}{*}{ Variable } & \multirow[b]{2}{*}{ Factor } & \multicolumn{4}{|c|}{ Bayesian } & \multirow{2}{*}{$\begin{array}{c}\text { Classical } \\
\text { Adjusted for age, } \\
\text { deprivation and period }\end{array}$} \\
\hline & & Adjusted for age & $\begin{array}{l}\text { Adjusted for } \\
\text { deprivation }\end{array}$ & $\begin{array}{l}\text { Adjusted for } \\
\text { period }\end{array}$ & $\begin{array}{c}\text { Adjusted for age, } \\
\text { deprivation and period }\end{array}$ & \\
\hline \multicolumn{7}{|l|}{ Fixed effects } \\
\hline \multirow[t]{4}{*}{ Age group } & $15-59$ years & 1.0 & & & 1.0 & 1.0 \\
\hline & $60-69$ years & $0.99(0.88,1.12)$ & & & $0.99(0.88,1.12)$ & $0.99(0.88,1.12)$ \\
\hline & $70-79$ years & $1.29(1.15,1.44)$ & & & $1.28(1.14,1.43)$ & $1.27(1.13,1.42)$ \\
\hline & $80+$ years & $2.34(2.08,2.64)$ & & & $2.27(2.02,2.55)$ & $2.23(1.98,2.51)$ \\
\hline \multirow[t]{5}{*}{ Deprivation quintile } & I (most affluent) & & 1.0 & & 1.0 & 1.0 \\
\hline & 2 & & $0.99(0.88,1.12)$ & & $0.98(0.88,1.09)$ & $0.97(0.87,1.08)$ \\
\hline & 3 & & $1.23(1.09,1.37)$ & & $1.21(1.08,1.34)$ & $1.21(1.09,1.34)$ \\
\hline & 4 & & $1.30(1.16,1.46)$ & & $1.27(1.14,1.41)$ & $1.30(1.18,1.44)$ \\
\hline & 5 (most deprived) & & $1.47(1.31,1.64)$ & & $1.43(1.28,1.58)$ & $1.45(1.32,1.60)$ \\
\hline \multirow[t]{2}{*}{ Period of diagnosis } & $1990-1995$ & & & 1.0 & 1.0 & 1.0 \\
\hline & $1996-2000$ & & & $0.64(0.60,0.68)$ & $0.67(0.64,0.71)$ & $0.67(0.63,0.7 I)$ \\
\hline
\end{tabular}

Table 3 Variance estimates for the random effects

\begin{tabular}{|c|c|c|c|c|c|}
\hline Variance & Unadjusted & $\begin{array}{c}\text { Adjusted for age } \\
\text { only }\end{array}$ & $\begin{array}{c}\text { Adjusted for } \\
\text { deprivation only }\end{array}$ & $\begin{array}{l}\text { Adjusted for } \\
\text { period only }\end{array}$ & $\begin{array}{l}\text { Adjusted for age, } \\
\text { deprivation and period }\end{array}$ \\
\hline \multicolumn{6}{|l|}{ PCT } \\
\hline Structured & $0.0286(0.0010,0.0510)$ & $0.0245(0.0013,0.0452)$ & $0.0218(0.0010,0.0395)$ & $0.0269(0.0015,0.0502)$ & $0.0177(0.003,0.032)$ \\
\hline Fraction structured & $0.70(0.02,0.99)$ & $0.69(0.04,0.99)$ & $0.72(0.03,0.99)$ & $0.67(0.04,0.99)$ & $0.74(0.14,0.98)$ \\
\hline DIC & 19464.8 & | 8996.1 & 19400.4 & 19236.2 & 18738 \\
\hline
\end{tabular}

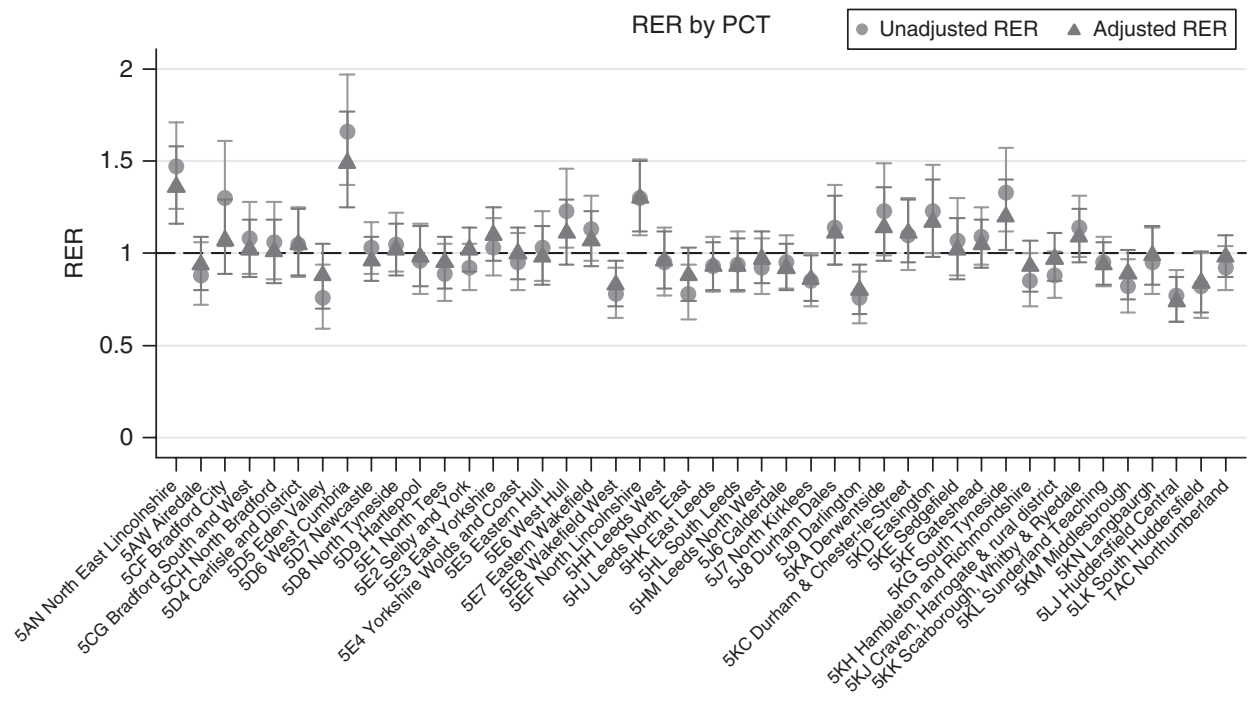

PCT

Figure 3 Relative excess risks (RER) by PCT.

was from 2 to 99\%. In each of the univariate models the percentage of the variance explained by the spatial effects was similar ranging from $67 \%$ for the model adjusted for period of diagnosis to $72 \%$ for the model adjusted for deprivation. In the fully adjusted model the fraction of the variation that was spatially structured was $74 \%$, and the width of the credible interval was narrower, from 14 to $98 \%$. The percentage of spatially structured variance in the fully adjusted model was greater than the unadjusted model and the univariate models suggesting that all three covariates might not be truly independent.
The maps in Figures 4 and 5 show the unadjusted and fully adjusted spatially smoothed RERs for each PCT. After adjusting for the covariates areas of high and low excess mortality were smoothed towards the mean, the number of PCTs with RER above 1.25 reduced from five to three whereas the number of PCTs with RER under 0.85 reduced from eight to four. After adjusting for the covariates area with higher excess risk of deaths were found in West Cumbria, North Lincolnshire and North East Lincolnshire whereas the areas with low excess risk of death were found in Huddersfield Central, South Huddersfield, Darlington and Wakefield West. 
Table 4 Descriptive statistics comparing adjusted RER for PCTs from Bayesian spatial and classical methods

\begin{tabular}{lcc} 
Measure & Bayesian RER & Classical RER \\
\hline Min & 0.74 & 0.44 \\
25th centile & 0.93 & 0.8 \\
Median & 0.99 & 0.99 \\
75 th centile & 1.08 & 1.31 \\
Max & 1.49 & 1.68 \\
Number of PCTs with RER $<0.85$ & 4 & 14 \\
Number of PCTs with RER $>1.25$ & 3 & 12 \\
\hline
\end{tabular}

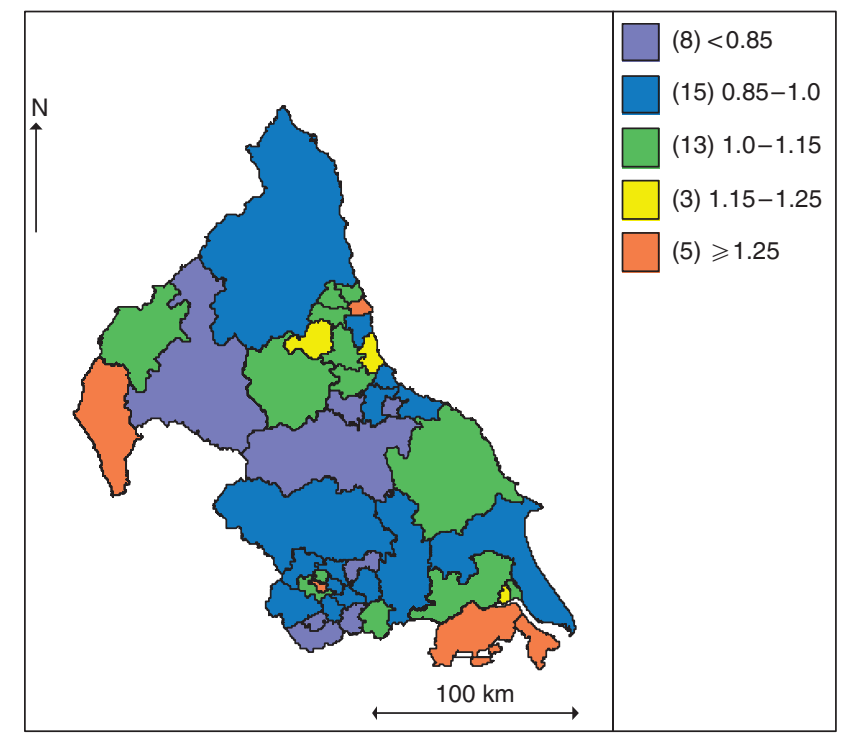

Figure 4 Map of unadjusted smoothed PCT spatial effects.

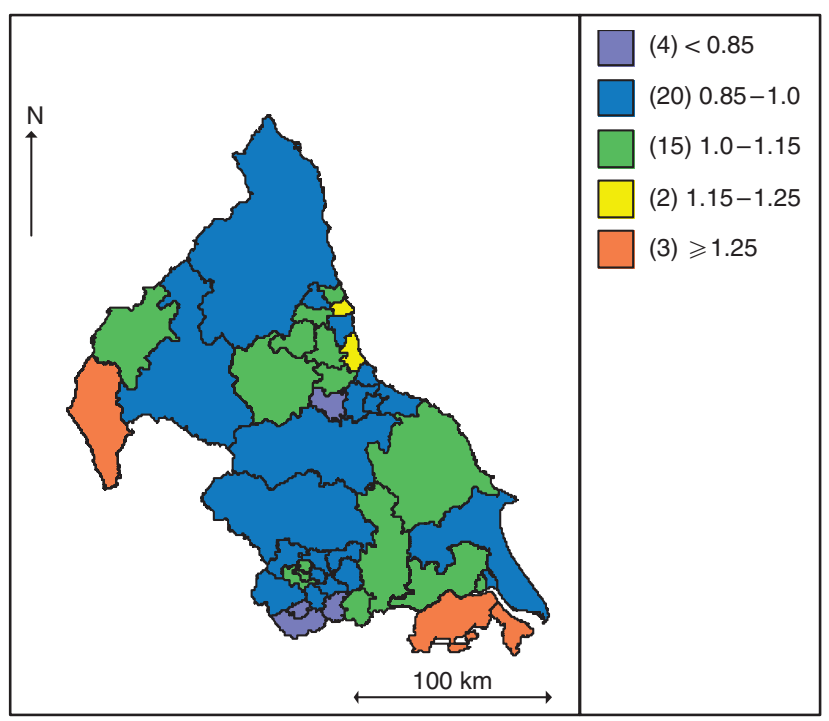

Figure 5 Map of fully adjusted smoothed PCT spatial effects.

\section{DISCUSSION}

Many studies have found that there is regional variation in prostate cancer survival (Farrow et al, 1996; Dickman et al, 1997; Post et al, 1998; EUROCARE, 2003; Yu et al, 2004; Berrino et al,
2007), we also found that there are differences in the survival patterns for PCTs in the NYCRIS region. The estimation of cancer survival at the PCT level can be problematic due to insufficient data to obtain robust estimates. In this paper, we have used Bayesian methods and adjusted for socio-demographic risk factors to produce survival estimates, in the form of relative excess risks of death, by PCT for prostate cancer in the NYCRIS region. By mapping the results we were able to identify the areas where high excess mortality was evident and where resources may be targeted to improve outcomes.

The results showed that all of the three included covariates had a significant association with excess mortality rates, men from more deprived areas, older age at diagnosis and diagnosed between 1990 and 1994 had higher excess mortality. However, the analysis has suggested that there may be some other risk factor that we have not accounted for that influences survival. Both stage of disease at diagnosis and treatment received will have an impact on patient survival.

Cancer stage is one of the most important predictors of survival. In New South Wales, Australia Bayesian models that adjusted for stage of disease were fitted and significant variation in prostate cancer survival between regions was found. ( $\mathrm{Yu}$ et al, 2004) The cancer registry does not have good quality data on stage for the Northern part of the NYCRIS region until the late 1990s, therefore, we are unable to adjust for this in our models and analysis of these variables over our study period is limited. However, some analysis on metastases rates across the PCTs based on data from 1998 and 1999 has been carried out (data not shown). The rate of metastases at presentation by PCT show that the three PCTs with higher excess risk of death had higher percentages of men presenting with metastases than the NCYRIS average whereas the four PCTs with lower excess risks of death had lower percentages of men with metastases. The differences in the proportions of men presenting with metastases across the PCTs may help explain some of the variation in survival that we have observed.

The stage at diagnosis is likely to have changed over time as the use of PSA testing has become more common in the UK (Brewster et al, 2000; Evans and Moller, 2003). This increase in PSA testing has lead to the diagnosis of many cancers earlier and that might not have been diagnosed in life. By diagnosing many more tumours earlier survival estimates will increase. We saw in our study the men diagnosed in the later time period had an excess risk of death $33 \%$ lower than men diagnosed in the earlier half of our study period.

There is also evidence to suggest that PSA testing is more common in affluent areas (Melia et al, 2004). Coleman et al (2004) found that in England and Wales inequality in deprivation increased over time and part of this increase in inequality could be because of increased PSA testing in the more affluent groups. There is likely to be a complex relationship between stage and deprivation and survival that is not accounted for in our models.

Treatment received for prostate cancer will also have a bearing on the survival of each individual. Currently in the UK there is no consensus on the best treatment for prostate cancer (NICE, 2002), and there are wide variations in the methods of treatment used in the UK. (Hanna et al, 2002; Payne and Gillatt, 2007) Geographical differences in treatment have been observed elsewhere (Bauvin et al, 2003; Krupski et al, 2005) and in our study region there is some evidence of differences in the treatment of prostate cancer received by cancer network. (NYCRIS, 2007) Looking at treatment data across the PCTs for 1998 and 1999 also shows that there is a wide variation in treatment modality by PCT (data not shown).

This study identified three areas with higher excess risks of death and four areas with lower risks of death after adjustment for age at diagnosis, deprivation and period of diagnosis. The age standardised incidence rates for the PCTs with higher excess risk were lower than the NYCRIS rate in 1990-1994 (one of these was statistically significantly lower) and two of the three PCTs had 
lower rates than the NYCRIS rate in 1995-1999 (one of these was statistically significantly lower). All the incidence rates for the four PCTs with lower excess risks were higher than the NYCRIS rate in 1990-1994 and this was statistically significantly higher in three. The rates were also higher for these PCTs in 1995-1999 and two of these were statistically significantly higher. The areas with higher rates may be diagnosing more cases due to increased use of PSA testing, therefore increasing survival rates as many of these cases will have early stage disease. Gavin et al (2004) found that there was a 100 -fold variation in PSA testing rates across general practises in Northern Ireland, we do not have data to assess the variation in levels of PSA testing across PCTs but differences in PSA testing by PCT will influence both incidence rates and survival rates by PCT.

We found that there was a significant deprivation gradient in the excess mortality; men from the most deprived areas had an excess mortality rate that was about $47 \%$ higher than that of men from the least deprived areas, this was attenuated slightly to $43 \%$ in the fully adjusted model. Deprivation was measured at the ward level, we do not have socio economic information at the individual level so have to use an area-based measure and should be aware that the results obtained for each area cannot be extrapolated to the individuals within that area.

In the fully adjusted model we found that the percentage of spatially structured variance was greater than the unadjusted models and the univariate models. This suggests that all three of the covariates in the model, age, deprivation quintile and period of diagnosis, might not be truly independent. There was a higher percentage of younger men diagnosed in 1995-1999 compared to 1990 - 1994, and there were more younger men diagnosed in affluent areas compared with the more deprived areas (data not shown).

We compared this spatial fraction after including different variables in the model to see how much of the geographical variation between PCTs can be explained by the risk factors. In the fully adjusted model $74 \%$ of the variation in survival is spatially structured which may indicate that there are some other spatially structured risk factors that we have not accounted for that may help explain the variation between the PCTs, for example differences in treatment or stage at diagnosis by PCT.

Many spatial analysis of incidence and mortality use data at a smaller geographical level than PCT, maybe ward or output area. Jarup et al (2002) used Bayesian methods to analyse spatial variations in prostate cancer incidence by ward in Great Britain and found there was no marked geographical variation in the risk of prostate cancer; however, this study used data from 1975 to 1991 before the use of PSA testing was more wide spread in the UK. This unit of analysis is not suitable for survival analysis as the models require a certain number of cases/deaths per interval. Also as treatment may influence survival and many patients will be treated within the same PCT, the PCT is a more logical geographic unit to use in survival analysis. However, we did carry a similar analysis based on ward of residence and found similar results as when we used the PCT data. There were clusters of wards with the highest excess risks of death in Cumbria, North Lincolnshire and North East Lincolnshire.

Bayesian methods are more frequently used to study spatial variation in cancer survival (Osnes and Aalen, 1999; Henderson et al, 2002; Yu et al, 2004) The use of Bayesian techniques to model cancer survival offers many advantages over the traditional methods. In particular, risk estimates in areas with small populations or low incidence rates, especially rural areas, are stabilised and shrunk towards local and global risk estimates. This improves the reliability and precision of the risk estimates. Therefore the chance of obtaining excessively high or low estimates due to the occurrence of sparse data is reduced. Other advantages of using a Bayesian approach are that it is relatively easy to fit complex models that allow adjustment for covariates when assessing geographical variation in survival and we can also obtain functions of the model parameters such as the relative contributions of the unstructured and structured spatial effects.

In our models we used an adjacency matrix that was based on the number of neighbours each PCT had. We used all of the PCTs in the NYCRIS region and some of these PCTs will border PCTs that are in a different cancer registry. In our study, these PCTs will only have recorded the PCTs that are in the NYCRIS region and this may introduce some bias into the results as these areas have neighbours missing. The credible interval for the estimates of the amount of variation that is due to spatially structured effects was rather wide; ranging from 2 to $99 \%$ in the unadjusted model, this decreased slightly in the fully adjusted model ranging from 14 to $98 \%$. The marginal spatial variance is inversely related to the total number of areas and in this study we only have 44 areas, the conditional spatial variance is also inversely proportional to the number of neighbours each area has and in this study we used quite large areas and they had relatively few neighbours, therefore the precision of the variance estimates would be smaller if we had used more smaller areas with more neighbours (such as wards).

The ability to detect areas where cancer survival is poor is a useful tool as it indicates where the policy to improve survival might be best targeted or where further investigation to understand the causes of the poor survival might be best carried out. As in this study it is important to be able to adjust for some common confounding variables such as age and deprivation. We feel that this methodology could be useful to apply to other cancer sites and geographic areas to gain a better understanding of regional variation in survival.

\section{REFERENCES}

Bauvin E, Soulié M, Ménégoz F, Macé-Lesec'h J, Buémi A, Velten M, Villers A, Grosclaude P (2003) Medical and non-medical determinants of prostate cancer management: a population-based study. Eur J Cancer 39: 2364-2371

Berrino F, De Angelis R, Sant M, Rosso S, Lasota MB, Coebergh JW, Santaquilani M (2007) Survival for eight major cancers and all cancers combined for European adults diagnosed in 1995-1999: results of the EUROCARE-4 study. Lancet Oncol 8: 773-783

Besag J, York J, Mollie A (1991) Bayesian image restoration, with two applications in spatial statistics (with discussion). Ann Inst Statist Math 43: $1-75$

Brewster DH, Fraser LA, Harris V, Black RJ (2000) Rising incidence of prostate cancer in Scotland: increased risk or increased detection. BJU Int 85: $463-473$

Coleman MP, Rachet B, Woods LM, Mintry E, Riga M, Cooper N, Quinn MJ, Brenner H, Esteve J (2004) Trends in Socioeconomic inequalities in cancer survival in England and Wales up to 2001. Br J Cancer 90: $1367-1373$

Dickman PW, Coviello E, Hills M (2008) Estimating and modelling relative survival. http://www.pauldickman.com/survival/strs.pdf (accessed February 2008)

Dickman PW, Gibberd RW, Hakulinen T (1997) Estimating potential savings in cancer deaths by eliminating regional and social class variation in cancer survival in the Nordic countries. I Epidemiol Community Health 51: 289-298

Dickman PW, Sloggett A, Hills M, Hakulinen T (2004) Regression models for relative survival. Stat $\mathrm{Med}$ 23: $51-64$

Ederer F, Axtell LM, Cutler SL (1961) The relative survival rate: a statistical methodology. Natl Cancer Inst Monogr 6: 101-121

Ellis L, Rachet B, Coleman MP (2007) Cancer survival indicators by cancer network: a methodological perspective. Health Stat $Q$ 36: $36-41$ 
Evans HS, Moller H (2003) Recent trends in prostate cancer incidence and mortality in Southeast England. Eur Urol 43: 337-341

Farrow DC, Samet JM, Hunt WC (1996) Regional variation in survival following the diagnosis of cancer. J Clin Epidemiol 49: 843-847

Gavin A, McCarron P, Middleton RJ, Savage G, Catney D, O'Reilly D, Keane PF, Murray LJ (2004) Evidence of prostate cancer screening in a UK region. BJU Int 93: $730-734$

Hakulinen T, Tenkanen L (1987) Regression analysis of relative survival rates. J R Statist Soc C 36: 309-317

Hanna CL, Mason MD, Donovan JL, Barber JP (2002) Clinical oncologists favour radical radiotherapy for localized prostate cancer: a questionnaire survey. BJU Int 90: $558-560$

Jarup L, Best N, Toledano M, Wakefield J, Elliot P (2002) Geographical epidemiology of prostate cancer in Great Britain. Int $J$ Cancer 97: 695-699

Henderson R, Shimakura S, Gorst D (2002) Modelling spatial variation in leukemia survival data. J Am Statist Ass 97: 965-972

Krupski TL, Kwan L, Afifi AA, Litwin MS (2005) Geographic and socioeconomic variation in the treatment of prostate cancer. J Clin Oncol 23: $7881-7888$

Melia J, Moss S, Johns L (2004) Rates of prostate-specific antigen testing in general practice in England and Wales in asymptomatic and symptomatic patients: a cross-sectional study. BJU Int 94: $51-56$

National Institute for Clinical Excellence (2002) Guidance on Cancer Services: improving outcomes in urological cancers - the manual. http:// www.nice.org.uk/nicemedia/pdf/Urological_Manual.pdf

Nobel M, Wright G, Dibben C, Smith GAN, McLenna D, Anttila C, Branes H, Mokhtar C, Nobel S, Avenell D, Gardner J, Covizzi I, Lloyd M (2004) Indices of Deprivation 2004. Report to the Office of the Deputy Prime Minister. Neighbourhood Renewal Unit: London
NYCRIS reference data 2004 (2004) www.nycris.org.uk (accessed February 2008)

NYCRIS (2007) Cancer in the 21st Century. NYCRIS Statistical Report for 2000 - 2004, www.nycris.org.uk

Osnes K, Aalen OO (1999) Spatial smoothing of cancer survival: a Bayesian approach. Stat Med 18: 2087-2099

Payne HA, Gillatt DA (2007) Differences and commonalities in the management of locally advanced prostate cancer: results from a survey of oncologists and urologists in the UK. BJU Int 99: 545-553

Pohar M, Stare J. (2006) Relative survival analysis in R. Comput Methods Programs in Biomed 81: 272-278

Post PN, Damhuis RAM, Van der Meyden APM, EUROCARE Working Group (1998) Variation in survival of patients with prostate cancer in Europe since 1978. Eur J Cancer 34: 2226-2231

Rachet B, Coleman MP (2004) Cancer Survival indicators for the National Health Service in England: exploration of alternative geographic units of analysis - the Primary Care Organisation and Strategic Health Authority. London School of Hygiene and Tropical Medicine 2004

Spiegelhalter DJ, Best NG, Carlin BP, Van der Linde A (2002) Bayesian measures of model complexity and fit (with Discussion). J R Statist Soc $B$ 64: $583-616$

Spiegelhalter DJ, Thomas A, Best NG, Lunn D (2004) BUGS: Bayesian Inference Using Gibbs Sampling, Version 1.4.1 Medical Research Council Biostatistics Unit, Cambridge University 2004, www.mrc-bsu.cam.ac.uk/ bugs/winbugs/manual14.pdf (accessed October 2008).

The EUROCARE Working Group (2003) EUROCARE-3: survival of cancer patients diagnosed 1990-1994 - results and commentary. Ann Oncol 14: v61 - v118

Yu XQ, O'Connell DL, Gibberd RW, Smith DP, Dickman PW, Amrstrong BK (2004) Estimating regional variation in caner survival: a tool for improving cancer care. Cancer Causes Control 15: 611-618 